\title{
Chronotaxic Systems: A New Class of Self-Sustained Nonautonomous Oscillators
}

\author{
Yevhen F. Suprunenko, Philip T. Clemson, and Aneta Stefanovska* \\ Department of Physics, Lancaster University, Lancaster LA1 4YB, United Kingdom
}

(Received 26 March 2013; published 8 July 2013)

\begin{abstract}
Nonautonomous oscillatory systems with stable amplitudes and time-varying frequencies have often been treated as stochastic, inappropriately. We therefore formulate them as a new class and discuss how they generate complex behavior. We show how to extract the underlying dynamics, and we demonstrate that it is simple and deterministic, thus paving the way for a diversity of new systems to be recognized as deterministic. They include complex and nonautonomous oscillatory systems in nature, both individually and in ensembles and networks.
\end{abstract}

DOI: 10.1103/PhysRevLett.111.024101

PACS numbers: 05.45.Xt, 05.65.+b, 05.90.+m, 89.75.Fb

Dynamical systems are generally seen as being either deterministic or stochastic. The advent of dynamical chaos several decades ago attracted much attention and illustrated that even complex dynamics can be deterministic. Dynamical systems can also be classified as autonomous (self-contained) or nonautonomous (subject to external influences). In reality, nonautonomous systems are the commoner, but they are far harder to treat. Until now they were mostly treated as stochastic or, alternatively, attempts were made to reformulate them as autonomous. Neither approach captures the characteristic properties of these systems.

In this Letter we propose a new class of nonautonomous systems and name them chronotaxic to characterize oscillatory systems with time-varying, but stable, amplitudes and frequencies. Nonautonomous oscillatory systems appear in various fields of research including neuroscience [1,2], cardiovascular dynamics [3], climate [4,5] and evolutionary science [6], as well as in complex systems and networks [7-9]. Although we are witnessing a rapid development of the theory of nonautonomous $[10,11]$ and random dynamical systems [12,13], nonautonomous oscillatory systems with stable but time-varying characteristic frequencies have to date not been addressed. When treated in an inverse approach such systems are usually considered as stochastic. In an attempt to cope with the problem, several methods for the inverse approach were introduced, including wavelet-based decomposition [14], bispectral analysis [15], harmonic detection [16] and phase coherence [17], and Bayesian-based inference [18] methods.

Consequently, time-dependent dynamics have been detected in different systems such as the cardiovascular system [3], mitochondrial oscillations [19], the brain [20,21], and surface state electrons on liquid helium [22]. Common to all these systems is that they are oscillatory, have stable amplitudes, and frequencies that are resistant to external perturbations. The variety of systems with these characteristics suggests that their dynamics are generated from a universal basis. To date, the description of stable oscillatory dynamics has been based on the model of autonomous self-sustained limit cycle oscillators [23]. While this model provides stable amplitude dynamics, frequencies of oscillations within this model can be easily changed even by weakest external perturbations.

The new class of nonautonomous oscillatory dynamical systems that we now propose account for such dynamics. The novelty of these systems is that not only are the amplitude dynamics stable but also the frequencies of the oscillations are time dependent and stable-i.e., their timedependent values cannot be easily altered by external perturbations. Their characteristics and ability to generate complex dynamics are demonstrated using a theoretical example and a recording from a biological system (the human heart). We also show how the properties of these systems allow the deterministic dynamics to be extracted.

The new class of systems is a subclass of nonautonomous systems whose definition is provided by a skewproduct flow [10,24,25] generated by unidirectionally coupled differential equations (also known as a masterslave configuration [7], or as drive and response systems [26])

$$
\dot{\mathbf{p}}=\mathbf{f}(\mathbf{p}), \quad \dot{\mathbf{x}}=\mathbf{g}(\mathbf{x}, \mathbf{p}),
$$

where $\mathbf{p} \in R^{n}, \mathbf{x} \in R^{m}$. The nonautonomous system $\mathbf{x}$ can be considered as driven by the system $\mathbf{p}$ in the sense that $\dot{\mathbf{x}}=\mathbf{g}(\mathbf{x}, \mathbf{p}(t))$ for any given solution $\mathbf{p}(t)$. Assuming $\mathbf{p}(t)$ is known, the solutions for $\mathbf{x}, \mathbf{x}\left(t, t_{0}, \mathbf{x}_{0}\right)$, depend on the actual time $t \in R$, the initial time $t_{0} \in R$, and the initial point $\mathbf{x}_{0} \in R^{m}$.

Now consider a system $\mathbf{x}$ from (1) with oscillatory and stable dynamics. First, in terms of amplitude of oscillations it means that the amplitude does not depend on initial conditions and resists external perturbations. In the phase space it means that all points converge to a closed isolated trajectory $\Gamma_{0}$, similarly to autonomous systems where $\Gamma_{0}$ is called a limit cycle [23]. However in autonomous systems $\Gamma_{0}$ has a peculiar property in that the frequency of the stable oscillations can be easily changed. This is because the position of a system on a limit cycle, described by a phase, is neither stable nor unstable-it is neutral. 
In contrast to autonomous systems, we introduce nonautonomous oscillatory systems $\mathbf{x}$ whose frequency of oscillations resists external perturbations but can simultaneously vary in time. The position of such systems on $\Gamma_{0}$ is attracted to a time-dependent point $\mathbf{x}^{A}(t)$. Therefore, the distinguishing feature of the nonautonomous system $\mathbf{x}$ is a unique steady state $\mathbf{x}^{A}(t)$. It moves along the limit cycle $\left(\mathbf{x}^{A}(t) \in \Gamma_{0}\right)$ with a certain angular velocity which determines the frequency of the oscillations. The time-dependent value of such frequency is determined by the system (1) and cannot be easily changed by influences on dynamics of $\mathbf{x}$ from processes external to (1). The steady state $\mathbf{x}^{A}(t)$ satisfies the condition of invariance,

$$
\mathbf{x}\left(t, t_{0}, \mathbf{x}^{A}\left(t_{0}\right)\right)=\mathbf{x}^{A}(t) .
$$

Also, $\mathbf{x}^{A}(t)$ attracts trajectories at actual time $t$,

$$
\lim _{t_{0} \rightarrow-\infty}\left|\mathbf{x}\left(t, t_{0}, \mathbf{x}_{0}\right)-\mathbf{x}^{A}(t)\right|=0 .
$$

Because of conditions (2) and (3) the point $\mathbf{x}^{A}(t)$ can be recognized as a pullback attractor [10,27].

Taking into account that oscillatory systems with stable amplitude dynamics and stable time-dependent frequencies play an important role in various branches of science $[3,19,20,22]$, we propose considering the nonautonomous systems $\mathbf{x}$ as a new class. These systems are defined by Eq. (1), by an attracting cycle $\Gamma_{0}$ and a time-dependent steady state $\mathbf{x}^{A}(t) \in \Gamma_{0}$. We name these systems chronotaxic (chronos-time, taxis-order) to emphasize their defining property, which is the ability to withstand perturbations to their time-varying parameters (frequencies). The motion of the point attractor $\mathbf{x}^{A}(t)$ along $\Gamma_{0}$ features as a special kind of limit cycle for nonautonomous systems-a chronotaxic limit cycle.

Properties of chronotaxic systems.-(i) Chronotaxic systems are dissipative dynamical systems representing open thermodynamical systems with an internal source of energy. Chronotaxic systems can sustain a stable oscillation amplitude against external perturbations. (ii) In contrast to autonomous limit-cycle oscillators, both the time-dependent phase and frequency of oscillations in chronotaxic systems are stable and can resist external perturbations.

Theoretical example and the inverse problem approach.-Consider the following 2D chronotaxic system whose limit cycle $\Gamma_{0}$ is a circle with the radius $r_{0}$ and whose phase and amplitude dynamics are separable. The system is described by a vector $\mathbf{x}=$ $\left(r_{\mathbf{x}} \cos \left(\alpha_{\mathbf{x}}\right), r_{\mathbf{x}} \sin \left(\alpha_{\mathbf{x}}\right)\right)$. The amplitude dynamics is described by

$$
\dot{r}_{\mathbf{x}}=\varepsilon_{r} r_{\mathbf{x}}\left(r_{0}-r_{\mathbf{x}}\right)
$$

where $\varepsilon_{r}$ is a constant. The phase dynamics is described by the equation for the phase $\alpha_{\mathrm{x}} \in R$,

$$
\dot{\alpha}_{\mathbf{x}}=\varepsilon \omega_{0}(t) \sin \left(\alpha_{\mathbf{x}}-\alpha_{\mathbf{p}}(t)\right)+\xi(t) .
$$

The coupling function $\varepsilon \omega_{0}(t) \sin \left(\alpha_{\mathbf{x}}-\alpha_{\mathbf{p}}(t)\right)$ explicitly depends on time, while the parameter $\varepsilon$ is constant and $\xi(t)$ represents external perturbation. The contribution from the $\mathbf{p}$ system (1) is represented by the phase $\alpha_{\mathbf{p}}(t)$, with dynamics given by $\dot{\alpha}_{\mathbf{p}}=\omega_{0}(t)$, where

$$
\omega_{0}(t)=0.3 \pi\left[1-\frac{\cos \left(\omega_{1} t\right)}{3}+\frac{\cos \left(\omega_{2} t\right)}{3}\right],
$$

with $\omega_{1}=0.0275 \pi \mathrm{rad} \mathrm{s}^{-1}, \omega_{2}=0.01325 \pi \mathrm{rad} \mathrm{s}^{-1}$.

The evolution of (4) and (5) with $\xi(t)=0$ and $\varepsilon_{r}=1$ is shown in Fig. 1(a). In such a case for $|\varepsilon| \geqslant 1$ and $\omega_{0}(t)>0$ all trajectories approach the pullback point attractor $\mathbf{x}^{A}(t)$
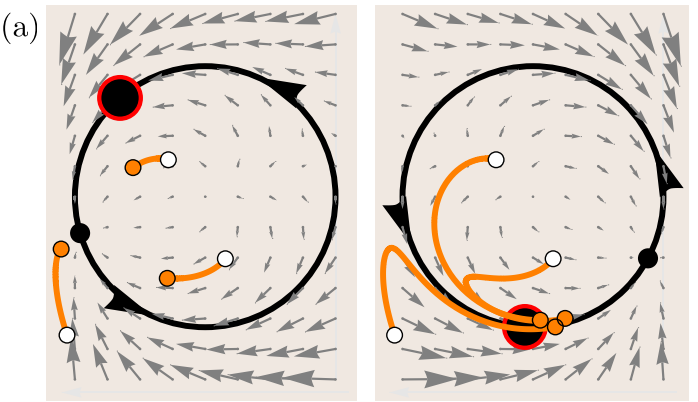

$2.5 \mathrm{~s}$
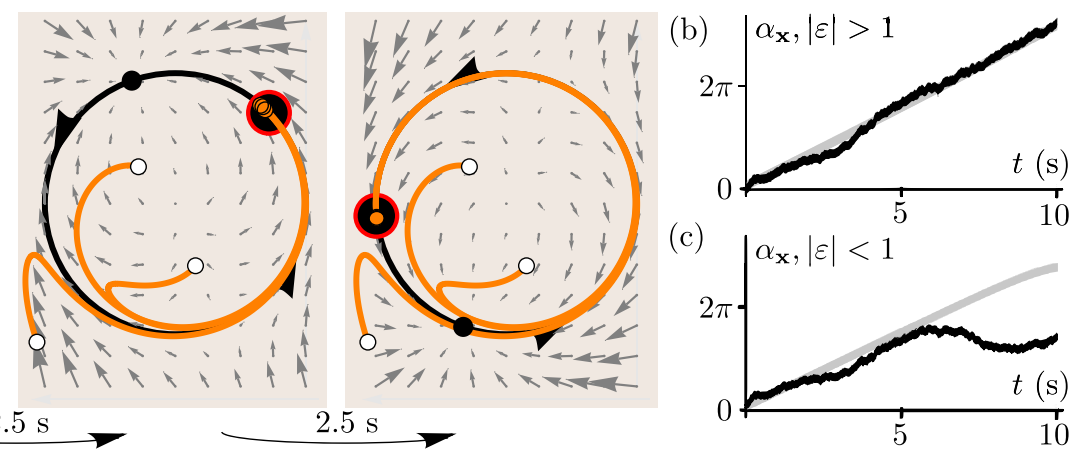

FIG. 1 (color online). (a) Time evolution of chronotaxic system (4) and (5) in the plane with polar coordinates $\left(\alpha_{\mathbf{x}}, r_{\mathbf{x}}\right)$ using $\varepsilon<-1$ and $\omega_{0}(t)>0$. Four phase portraits separated by $2.5 \mathrm{~s}$ time intervals are shown. The time-dependent point attractor or steady state $\left(\alpha_{\mathbf{x}}^{A}(t), r_{0}\right)$ (large black disk in red circle) moves along a limit cycle (black circle). Trajectories (orange) from different initial conditions (white dots) approach the point attractor and then move together with it. This occurs because $\alpha_{\mathbf{x}}$ is coupled to a moving point $\left(\alpha_{\mathbf{p}}(t), r_{0}\right)$ (small black disk) which results from a nonautonomous contribution. The coupling determines instantaneous velocities (gray arrows) of the system and the angular distance $\alpha_{\mathbf{p}}(t)-\alpha_{\mathbf{x}}^{A}(t)=\arcsin (1 /|\varepsilon|)$. (b) The position (phase) $\alpha_{\mathbf{x}}$ of the chronotaxic system on the limit cycle when unperturbed (straight gray line) and perturbed (noisy black line). (c) A moving point attractor does not exist on a limit cycle, the perturbed phase strongly deviates from the unperturbed phase. 
[large black disk in red circle in Fig. 1(a)] which satisfies conditions (2) and (3). Its polar coordinates $\left(\alpha_{\mathbf{x}}^{A}(t), r_{0}\right)$ can be found analytically: $\alpha_{\mathbf{x}}^{A}(t)=\alpha_{\mathbf{p}}(t)-\arcsin (1 /|\varepsilon|)+$ $0.5 \pi(1+\operatorname{sign}(\varepsilon))+2 \pi k$, where $k$ is an arbitrary integer number. In the rotating reference frame where $\left(\alpha_{\mathbf{p}}(t), r_{0}\right)$ and $\left(\alpha_{\mathbf{x}}^{A}(t), r_{0}\right)$ are stationary all trajectories of the system (4) and (5) approach the steady state $\left(\alpha_{\mathbf{x}}^{A}(t), r_{0}\right)$. Now consider the function $\xi(t)$ as white Gaussian noise $\left(\langle\xi(t)\rangle=0,\left\langle\xi(t) \xi\left(t^{\prime}\right)\right\rangle=\sigma^{2} \delta\left(t-t^{\prime}\right)\right)$. The moving point attractor provides stability in the phase $\alpha_{\mathbf{x}}(t)$. Perturbations by external additive noise $\xi(t)$ therefore do not cause $\alpha_{\mathbf{x}}(t)$ to drift from the attractor, as shown in Fig. 1(b). However, when the moving point attractor is absent, $|\varepsilon|<1$, the phase $\alpha_{\mathbf{x}}$ strongly deviates from the unperturbed phase, Fig. 1(c).

Assuming that the amplitude of oscillations is more resistant against external perturbations than the frequency of oscillations $\left(\varepsilon_{r} \gg|\varepsilon|\right)$, and consequently assuming deviations of the system $\mathbf{x}$ from a limit cycle are negligible $\left(\varepsilon_{r} \gg \sigma\right)$ we focus only on phase dynamics. Considering the system (5) we analyze its time series and present results in Fig. 2(a) using $\varepsilon=1.1$ and $\sigma=0.075$. Results obtained by the Fourier transform and time-delay embedding [28-30] are shown in Figs. 2(b) and 2(c), respectively. However, these methods are unable to trace timedependent behavior and therefore do not identify stable oscillatory dynamics. A reconstruction of dynamics using a time-delay embedding results in time-dependent components being considered as extra dimensions of an autonomous system [31]. Therefore, the embedded trajectories gradually fill low-dimensional phase space and the attractor is not reconstructed. However, when only a small part of the trajectories are considered, a time-dependent cycle emerges from the system without noise $(\xi(t)=0)$, Fig. 2(d), reflecting oscillations with time-dependent frequencies on the cycle $\Gamma_{0}$.

Different modes of the dynamics analyzed using the wavelet transform are shown in Figs. 2(e)-2(g). The unperturbed dynamics of the system (5) are shown in Fig. 2(e). The line in the wavelet transform corresponds to the frequency $\omega_{0}(t)$, Eq. (6). The dynamics can become complex and stochastic when the system is strongly perturbed, Fig. 2(f), resulting in a large amount of phase slips, which means that the difference $\left|\alpha_{\mathbf{x}}-\alpha_{\mathbf{x}}^{A}\right|$ increases by a multiple of $2 \pi$. The number of phase slips decreases when the strength of the coupling function increases, as illustrated in Fig. 2(g). Nevertheless, the dynamics may look totally stochastic and it can be difficult to extract the underlying deterministic dynamics of the system. In chronotaxic systems, however, it is possible to extract the unperturbed dynamics determined by motion of the point attractor. Such dynamics correspond to a dominant, time-varying oscillatory component in the wavelet transform, as can be seen in Fig. 2(g). This extraction and separation of these dynamics results in a reduction in the complexity of chronotaxic systems.

Chronotaxic systems are expected to have various types of behavior because phase and amplitude dynamics may not be separable. The attraction to $\Gamma_{0}$ may not necessarily be stronger than the attraction to $\mathbf{x}^{A}$ along $\Gamma_{0}$. In the general case chronotaxic systems can have time-dependent amplitude dynamics, such that the point attractor moves along the time-dependent attracting circle $\Gamma_{0}(t)$.
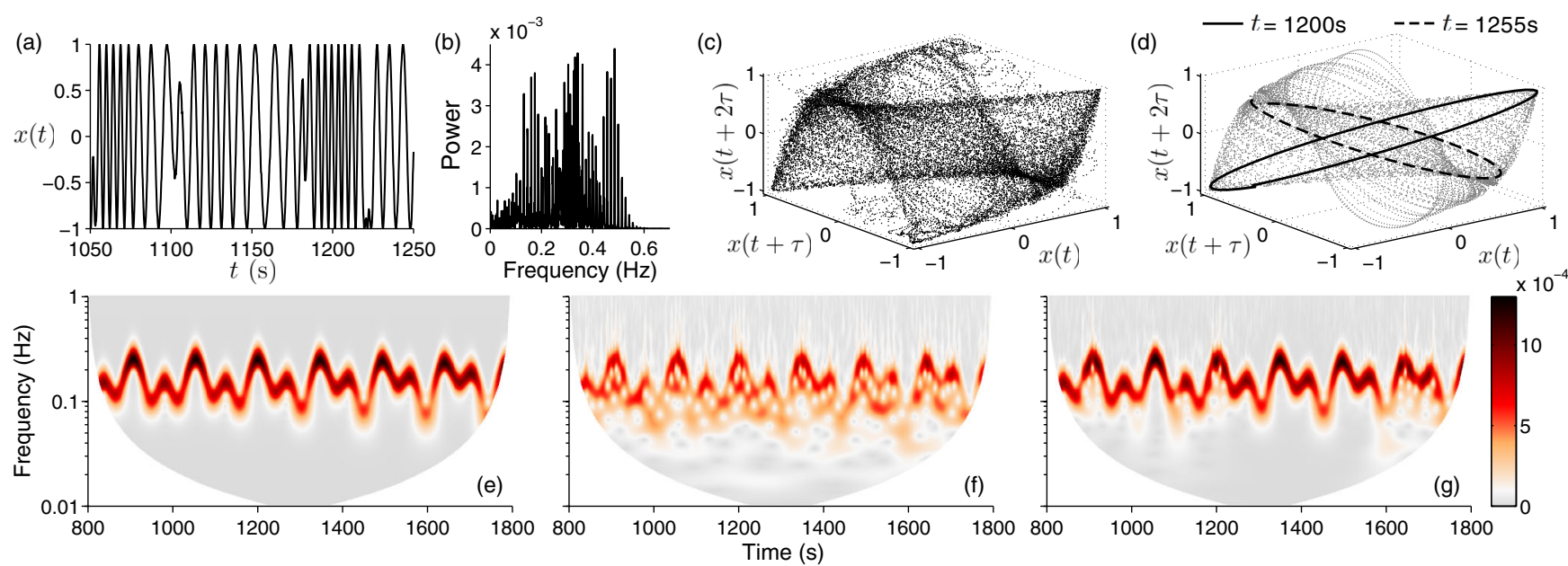

FIG. 2 (color online). Different domains of analysis of a signal $x(t)=\cos \left(\alpha_{\mathbf{x}}(t)\right)$ from system (5). Change in frequency, $\dot{\alpha}_{\mathbf{x}}(t)$, and phase slips as seen in (a) the time domain, (b) Fourier power spectrum and (c) phase space obtained by time-delay embedding [28,29] for $\varepsilon=1$.1. (d) The embedding of the signal from the unperturbed $(\xi(t)=0)$ system (5) with $\tau=1.9 \mathrm{~s}$, which corresponds to the first minimum of the mutual information [30]. Two small parts of the embedded trajectory during time intervals $\left(t, t+2 \pi / \omega_{0}(t)\right)$ reveal a time-dependent cycle. (e) Wavelet transform of the signal from the unperturbed system (5) with $\varepsilon \geq 1$. (f) When a point attractor does not exist $(\varepsilon=0.9)$ continuous phase slips occur for the perturbed system. (g) Phase slips also occur for the perturbed system at the bifurcation point $(\varepsilon=1)$ but the attractor exists and can be reconstructed. When $\varepsilon$ increases in time, phase slips become less frequent and almost disappear around $t=1400 \mathrm{~s}$ where $\varepsilon(t)=1+5 \omega_{0}^{-1}(t) \exp \left[-(t-1400)^{2} / 5000\right]$. 
Human heart as a chronotaxic system.-We now illustrate that the human heart can be considered as a chronotaxic system. We conducted an experiment on a healthy young subject where respiration was paced with the frequency $\omega_{0}(t)$, Eq. (6), as seen in Fig. 3(a). The respiration was measured using a stretch-sensitive belt around the chest and an electrocardiogram was recorded simultaneously. The instantaneous heart rate, also known as heart rate variability (HRV), was calculated from intervals between $R$ peaks in the electrocardiogram. Linear interpolation was applied to give a constant sampling frequency of $10 \mathrm{~Hz}$. Results of analysis of HRV in different domains are shown in Figs. 3(b)-3(d). The wavelet transform of the detrended HRV, Fig. 3(b), reveals how the heart rate follows the frequency of respiration. The trend is defined using a $25 \mathrm{~s}$ moving average and subtracted to remove the influence of fluctuations on longer time scales. The dominant line in Fig. 3(b) is discontinuous, with features similar to the phase slips seen in the theoretical example presented above, Fig. 2(g). However, in the phase space of the time-delay embedded detrended HRV signal [Fig. 3(c)], the dynamics appear entirely random and stochastic, reflecting the long-standing perception of heart dynamics.

We now search for signatures of chronotaxicity, i.e., a time-dependent point attractor moving on a limit cycle. We assume the randomness seen in phase space results from the amplitude dynamics hiding the limit cycle. This possibility is supported by the fact that we see one dominant but discontinuous line in the wavelet transform, Fig. 3(b). Comparing with the theoretical example, Fig. 2(g), we conclude that these discontinuities are due to phase slips which signify a presence of a point attractor. Therefore, in order to obtain the dynamics of the point attractor we extract the phase of the dominant component of HRV and embed it as shown in Fig. 3(d). It is clear that dynamics in the embedding space are governed by a time-dependent cycle [32], similarly to what we observed earlier in Fig. 2(d). These findings illustrate that the heart can be regarded as a chronotaxic system. Furthermore, this clearly shows the benefits of describing a system as chronotaxic; namely, the complexity of its dynamics is reduced when the deterministic part is extracted from other contributions.

Summary and conclusions. - We have introduced a new class of nonautonomous oscillatory systems and named them chronotaxic to encompass their defining propertythe ability to sustain stability in the amplitude and timedependent frequencies of oscillations under continuous perturbation. This ability is generated by a time-dependent point attractor or steady state which moves along the attracting cycle in phase space. We formulated properties of chronotaxic systems and illustrated them using a theoretical example. We also showed that chronotaxicity could be identified in a physiological system. Chronotaxic systems have many potential applications especially in modeling of complex systems, networks, and ensembles of oscillators when not all parts of the dynamics are directly observed. They are essential for effective modeling of living systems because they have inherently stable but time-varying characteristic frequencies. In addition, chronotaxic systems provide a new mechanism of obtaining complex dynamics from stable deterministic dynamics. While the dynamics under external perturbation may look stochastic, we have demonstrated that the underlying deterministic motion of the point attractor can be extracted. The work is therefore changing the perception of what is seen as the deterministic universe. Hence, we expect many chronotaxic systems to be discovered in nature, leading to various applications and an improved understanding of their dynamical behavior.
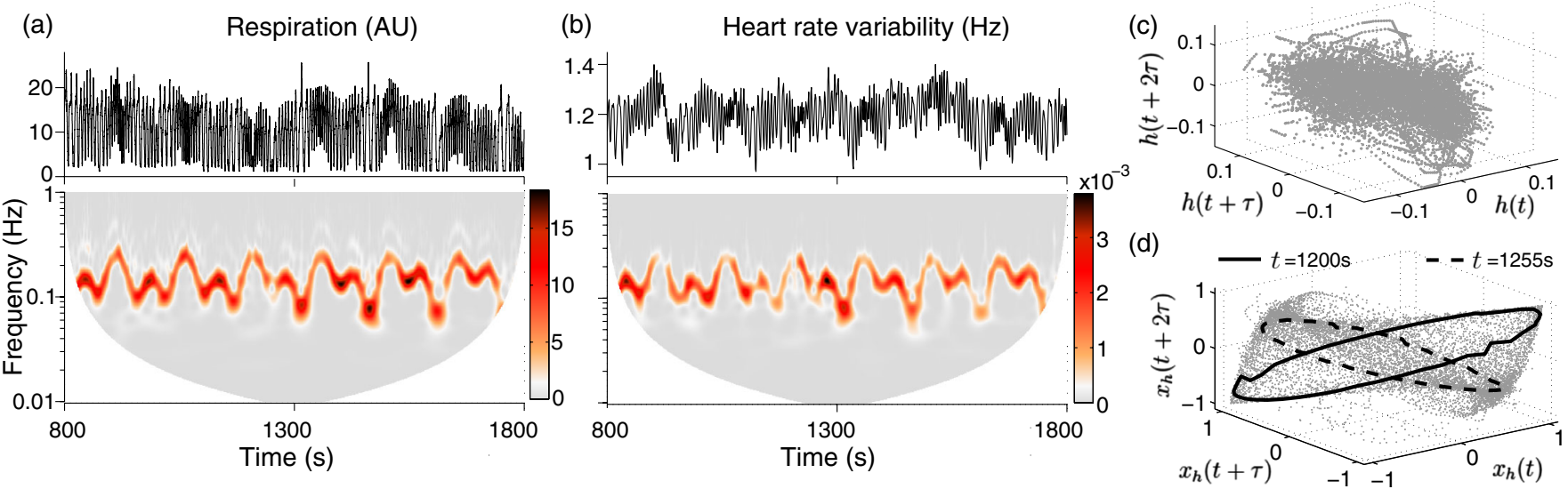

FIG. 3 (color online). Analysis of (a) respiration and (b) heart rate variability (HRV) recorded while respiration was paced. The top plots in (a) and (b) show the raw time series. Wavelet transforms of the detrended time series are shown at the bottom. The phase space plots (c) and (d) show the time-delay embedded time series of (c) detrended HRV, $h(t)$, with time-delay $\tau=1.9 \mathrm{~s}$, and (d) $x_{h}(t)=\cos \left(\alpha_{h}(t)\right)$ with time-delay $\tau=1.5 \mathrm{~s}$, where $\alpha_{h}(t)$ is a phase of $h(t)$ which was reconstructed using a method based on the synchrosqueezed wavelet transform [14]. 
Our grateful thanks are due to D. Burton, V. Cheianov, N. Drummond, J. Gratus, M. Horvat, D. Iatsenko, P. Kloeden, P. V.E. McClintock, M. Rasmussen, T. Stankovski, and R. Tindjong for valuable comments on the manuscript. This work was supported by the Engineering and Physical Sciences Research Council (UK) (Grant No. EP/100999X1).

*aneta@lancaster.ac.uk

[1] K. Saermark, Y. Ashkenazy, J. Levitan, and M. Lewkowicz, Physica (Amsterdam) 236A, 363 (1997).

[2] C. J. Stam, Clin. Neurophysiol. 116, 2266 (2005).

[3] Y. Shiogai, A. Stefanovska, and P. V. E. McClintock, Phys. Rep. 488, 51 (2010).

[4] C.S. Bretherton, M. Widmann, V.P. Dymnikov, J. M. Wallace, and I. Blade, J. Clim. 12, 1990 (1999).

[5] M. D. Chekroun, E. Simonnet, and M. Ghil, Physica (Amsterdam) 240D, 1685 (2011).

[6] P. M. C. de Oliveira, Theory Biosci. 120, 1 (2001).

[7] H. Haken, Synergetics: An Introduction and Advanced Topics (Springer, Berlin, 2004).

[8] Y. Kuramoto, Chemical Oscillations, Waves, and Turbulence (Springer-Verlag, Berlin, 1984).

[9] S. H. Strogatz, Nature (London) 410, 268 (2001).

[10] P.E. Kloeden and M. Rasmussen, Nonautonomous Dynamical Systems (American Mathematical Society, Providence, 2011).

[11] M. I. Vishik and V. V. Chepyzhov, Mathematical Notes 51, 622 (1992).

[12] H. Crauel and F. Flandoli, Probab. Theory Relat. Fields 100, 365 (1994).

[13] F. J. Romeiras, C. Grebogi, and E. Ott, Phys. Rev. A 41, 784 (1990).

[14] I. Daubechies, J. Lu, and H. Wu, Appl. Comput. Harmon. Anal. 30, 243 (2011).

[15] J. Jamšek, M. Paluš, and A. Stefanovska, Phys. Rev. E 81, 036207 (2010).
[16] L. W. Sheppard, A. Stefanovska, and P. V. E. McClintock, Phys. Rev. E 83, 016206 (2011).

[17] L. W. Sheppard, A. Stefanovska, and P. V. E. McClintock, Phys. Rev. E 85, 046205 (2012).

[18] T. Stankovski, A. Duggento, P. V.E. McClintock, and A. Stefanovska, Phys. Rev. Lett. 109, 024101 (2012).

[19] F. T. Kurz, M.A. Aon, B. O'Rourke, and A. A. Armoundas, Am. J. Physiol.: Heart Circ. Physiol. 299, H1736 (2010).

[20] D. Rudrauf, A. Douiri, C. Kovach, J.P. Lachaux, D. Cosmelli, M. Chavez, C. Adam, B. Renault, J. Martinerie, and M.L. Van Quyen, NeuroImage 31, 209 (2006).

[21] K. Friston, Entropy 14, 2100 (2012).

[22] D. Konstantinov, A. D. Chepelianskii, and K. Kono, J. Phys. Soc. Jpn. 81, 093601 (2012).

[23] A. A. Andronov, A. A. Vitt, and S. E. Khaikin, The Theory of Oscillators (Pergamon Press, Oxford, 1966).

[24] P.E. Kloeden and V.S. Kozyakin, Discrete Contin. Dyn. Syst. 7, 883 (2001).

[25] P.E. Kloeden, C. Potzsche, and M. Rasmussen, J. Difference Eq. Appl. 18, 693 (2012).

[26] L. Kocarev and U. Parlitz, Phys. Rev. Lett. 76, 1816 (1996).

[27] P. E. Kloeden, J. Difference Eq. Appl. 6, 33 (2000).

[28] F. Takens, in Lecture Notes in Mathematics, edited by D. A. Rand and L. S. Young (Springer, New York, 1981), Vol. 898.

[29] R. Mãne, in Lecture Notes in Mathematics, edited by D. A. Rand and L. S. Young (Springer, New York, 1981), Vol. 898.

[30] A. M. Fraser and H. L. Swinney, Phys. Rev. A 33, 1134 (1986).

[31] P. Clemson and A. Stefanovska, AIP Conf. Proc. 1468, 69 (2012).

[32] The signature of chronotaxicity can be investigated further by extracting the coupling and unperturbed phase using a method such as Bayesian-based inference $[18,33]$.

[33] A. Duggento, T. Stankovski, P. V.E. McClintock, and A. Stefanovska, Phys. Rev. E 86, 061126 (2012). 